\title{
Molecular dynamics calculations of the electrochemical properties of electrolyte systems between charged electrodes
}

\author{
Richard L. Rowley \\ rowley@byu.edu \\ Paul S. Crozier \\ Douglas Henderson
}

Follow this and additional works at: https://scholarsarchive.byu.edu/facpub

Part of the Chemical Engineering Commons

\section{Original Publication Citation}

P.S. Crozier, R.L. Rowley, and D. Henderson, â€œMolecular dynamics calculations of the electrochemical properties of electrolyte systems between charged electrodesâ€, J. Chem.

Phys. 113, 922 (2)

\section{BYU ScholarsArchive Citation}

Rowley, Richard L.; Crozier, Paul S.; and Henderson, Douglas, "Molecular dynamics calculations of the electrochemical properties of electrolyte systems between charged electrodes" (2000). Faculty Publications. 585.

https://scholarsarchive.byu.edu/facpub/585

This Peer-Reviewed Article is brought to you for free and open access by BYU ScholarsArchive. It has been accepted for inclusion in Faculty Publications by an authorized administrator of BYU ScholarsArchive. For more information, please contact ellen_amatangelo@byu.edu. 


\title{
Molecular dynamics calculations of the electrochemical properties of electrolyte systems between charged electrodes
}

\author{
Paul S. Crozier ${ }^{\text {a) }}$ and Richard L. Rowley ${ }^{\text {b) }}$ \\ Department of Chemical Engineering, Brigham Young University, Provo, Utah 84602-4100 \\ Douglas Henderson ${ }^{\text {) }}$ \\ Department of Chemistry and Biochemistry, Brigham Young University, Provo, Utah 84602-5700
}

(Received 27 June 2000; accepted 1 September 2000)

\begin{abstract}
We investigate the interfacial electrochemical properties of an aqueous electrolyte solution with discrete water molecules in slab geometry between charged atomistic electrodes. Long-range intermolecular Coulombic interactions are calculated using the particle-particle-particle-mesh method with a modification to account for the slab geometry. Density distribution profiles and potential drops across the double layer are given for $0,0.25$, and $1 \mathrm{M}$ aqueous electrolyte solutions each at $0, \pm 0.1, \pm 0.2$, and $\pm 0.3 \mathrm{C} / \mathrm{m}^{2}$ electrode surface charges. Results are compared qualitatively with experimental $\mathrm{x}$-ray scattering findings, other computer simulation results, and traditional electrochemistry theory. The interfacial fluid structure characteristics are generally in good qualitative agreement with the conclusions obtained in some integral equation theories and in the experimental $\mathrm{x}$-ray study. The potential in the simulations shows an oscillatory behavior near the electrode, which theories that do not include the molecular nature of water cannot reproduce for the given conditions. Surprisingly, the results also show that the water structure near the electrode is dominated by the charge on the electrode and is fairly insensitive to the ion concentrations. Except at large electrode charge, the potential drop across the double layer does not depend significantly upon the concentration of the ions. (C) 2000 American Institute of Physics.
\end{abstract}

[S0021-9606(00)51344-0]

\section{INTRODUCTION}

The ability to model correctly electrochemical processes at interfaces is a key to our understanding of numerous very important chemical and biological processes. Especially of interest are aqueous solutions in contact with charged surfaces, which can be found in such diverse fields as molecular biology, electrochemistry, and heterogeneous catalysis.

Although unable to model quantum mechanical effects, such as electron transfer, classical molecular dynamics is the most valuable tool available for the elucidation of important interfacial processes. Such simulations provide realistic representations of interfacial electrochemistry processes, limited primarily by molecular model accuracy and computational demands.

In order to save time, many interfacial electrochemistry molecular dynamics researchers neglect long-range Coulombic interactions while admitting the vital role that these play. ${ }^{1-5}$ Other researchers have employed costly Ewald summation approximations for long-range interactions. ${ }^{6-9} \mathrm{Re}-$ cently, mesh routines have been developed, ${ }^{10-15}$ which present a more efficient way to perform these calculations. Of the mesh routines, the particle-particle-particle-mesh $\left(\mathrm{P}^{3} \mathrm{M}\right)$ routine developed by Hockney and Eastwood ${ }^{10}$ has been shown to be the most efficient for a given accuracy level. ${ }^{14,15}$ In this work, we modify $\mathrm{P}^{3} \mathrm{M}$ for application to slab geometry, as suggested by Pollock and Glosli ${ }^{16}$ and

\footnotetext{
${ }^{a)}$ Electronic mail: crozierp@et.byu.edu

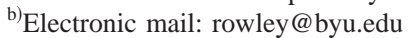

${ }^{c}$ Electronic mail: doug@ @uey.byu.edu
}

similar to the 3D Ewald summation modification used by Yeh and Berkowitz. ${ }^{8}$ We show that this implementation of the slab- "corrected" $\mathrm{P}^{3} \mathrm{M}$ method, or $\mathrm{P}^{3} \mathrm{MC}$ as we shall call it, gives results in very good agreement with regular Ewald summation methods, yet at a fraction of the cost.

Once we have established the validity of the $\mathrm{P}^{3} \mathrm{MC}$ methodology, we present simulation results obtained using this method dealing with the effect of electrode charge and ion concentration on the potential drop across the double layer of an aqueous electrolyte solution at a solid atomistic surface. We also note the similarity of our results with the experimental force measurements of Israelachvili et al. ${ }^{17}$ and the x-ray scattering findings of Toney et al. ${ }^{18}$ In addition, we compare our findings with the predictions of traditional interfacial electrochemistry theories, as well as with the findings from other simulation work.

\section{PMC IMPLEMENTATION}

Many methods for approximating long-range Coulombic interactions have been devised, including the charged sheets method, ${ }^{19,20}$ the modified reaction field, ${ }^{21-24}$ Ewald sums, ${ }^{6-9,25-27}$ the fast multipole method, ${ }^{16,28-30}$ and mesh methods. ${ }^{10-15}$ Recently, Crozier et al. ${ }^{9}$ found that the charged sheets method is inadequate for correctly modeling strong long-range Coulombic interactions for systems with discrete solvent molecules. Ewald summations appear to be adequate, yet computationally expensive, and multipole methods are even more costly for systems of a reasonable size. ${ }^{16}$ Mesh methods, particularly $\mathrm{P}^{3} \mathrm{M}$, present the most flexible and reliable alternative. ${ }^{14-16}$ Deserno and Holm give 
an excellent analysis of the various mesh methods, ${ }^{14}$ along with useful recommendations for $\mathrm{P}^{3} \mathrm{M}$ implementation. ${ }^{15} \mathrm{We}$ have followed their recommendations and adopted their notation in the following.

We use Fourier space, or $i \mathbf{k}$, differentiation to calculate the intermolecular forces from the electrostatic energies, and a seventh-order assignment scheme $(P=7)$ to assign the charges to the mesh. Calculation of the optimal influence function, $\hat{G}_{\text {opt }}$, of Hockney and Eastwood ${ }^{10}$ is done at the beginning of each run and therefore presents no additional run-time overhead. The cutoff for the Lennard-Jones (LJ) interactions, and the real-space Coulombic interactions is set at $r_{\text {max }}=10 \AA$, and the mesh size is set at $16 \times 16 \times 64$ in the $x, y$, and $z$ directions, respectively. With a corresponding fixed box size of $25.5 \AA \times 25.5 \AA \times 85.1232 \AA$, and the total number of ions and water molecules fixed at 1000 the optimum value of $\alpha$ is estimated to be 0.3007 . We estimate the average error in our force calculations to be on the order of $10^{-5}$ dimensionless force units, according to the estimation method and dimensionless force units of Deserno and Holm. ${ }^{14}$ Although not as precise as an Ewald sum, the amount of error introduced by this implementation of $\mathrm{P}^{3} \mathrm{M}$ is very reasonable. Extreme accuracy is not needed, especially when considering other possible sources of error in the MD simulation, such as the use of a randomly fluctuating thermostat, or a discrete time step (e.g., $2.5 \mathrm{fs}$ ).

In order to account for the slab geometry, we include an empty space, equal to the size of the fluid-occupied space, between repeating slabs of fluid, and a correction term to damp out interslab interactions. With the implementation of this correction, the $3 \mathrm{D}$, periodically repeating simulation closely approximates the simulation of an isolated 2D slab of finite thickness, as shown by Yeh and Berkowitz. ${ }^{8}$

To confirm the reliability of our $\mathrm{P}^{3} \mathrm{MC}$ implementation, we compare calculated forces with those calculated using the corrected 3D Ewald (EW3DC) method. ${ }^{8}$ In particular, we calculate the force between two oppositely charged particles along with their periodic images in slab geometry. For easy comparison, we consider the same case as presented in Refs. 7-9 $\left(L=L_{x}=L_{y}=18 \AA\right.$ and $\left.L_{z}=90 \AA\right)$. As seen in Figs. 1 and 2, the $\mathrm{P}^{3} \mathrm{MC}$ and EW3DC calculations are visually indistinguishable. For Fig. 1, the difference between the two calculations was less than $0.013 \%$ in all cases, with an average difference of $0.002 \%$. Even though the deviation was up to $1 \%$ in the case of Fig. 2, percent deviation is less meaningful here, since the absolute value of the force approaches zero. The absolute difference between the two calculations on the scale of Fig. 2 was always less than $10^{-4}$, which represents a very small amount of error. We also performed a test case simulation of the smaller system size as described in Ref. 9, and we found the ion and water density profiles to be in good agreement with the EW3DC results presented there.

With the chosen mesh parameters, our $\mathrm{P}^{3} \mathrm{M}$ algorithm was approximately four times faster than the corresponding Ewald algorithm. It should be noted that the flexibility of $\mathrm{P}^{3} \mathrm{M}$ allows for more exact force calculations if desired. The use of a tighter mesh would clearly be more precise, but more CPU intensive.

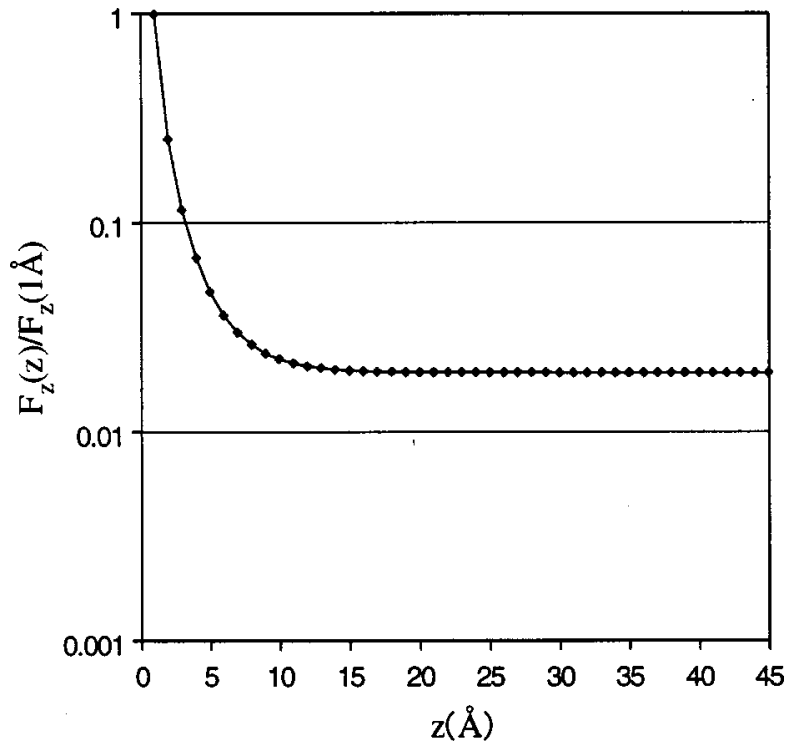

FIG. 1. Comparison of the force acting between two oppositely charged point charges in a two-dimensional periodic system as calculated using EW3DC (line), and $\mathrm{P}^{3} \mathrm{MC}$ (diamonds). The scale is chosen for comparison with Fig. 8 of Ref. 7, Fig. 4 of Ref. 8, and Fig. 3 of Ref. 9.

\section{SIMULATION DETAILS}

Simulations were performed at three ion concentrations, $0,0.25$, and $1 \mathrm{M}$, each at four electrode surface charges, 0 , $\pm 0.1, \pm 0.2$, and $\pm 0.3 \mathrm{C} / \mathrm{m}^{2}$. Previous studies that included discrete solvent molecules considered only higher ionic concentrations. In all cases, an equal number of monovalent anions and cations of equal size were used, and opposite walls were given equal and opposite charges for an electrostatically neutral system. The well-known SPC/E model of water was used, ${ }^{31}$ and the mobile ions were given LJ parameters identical to the SPC/E oxygen-oxygen parameters. Initially, the water molecules and ions were randomly distributed

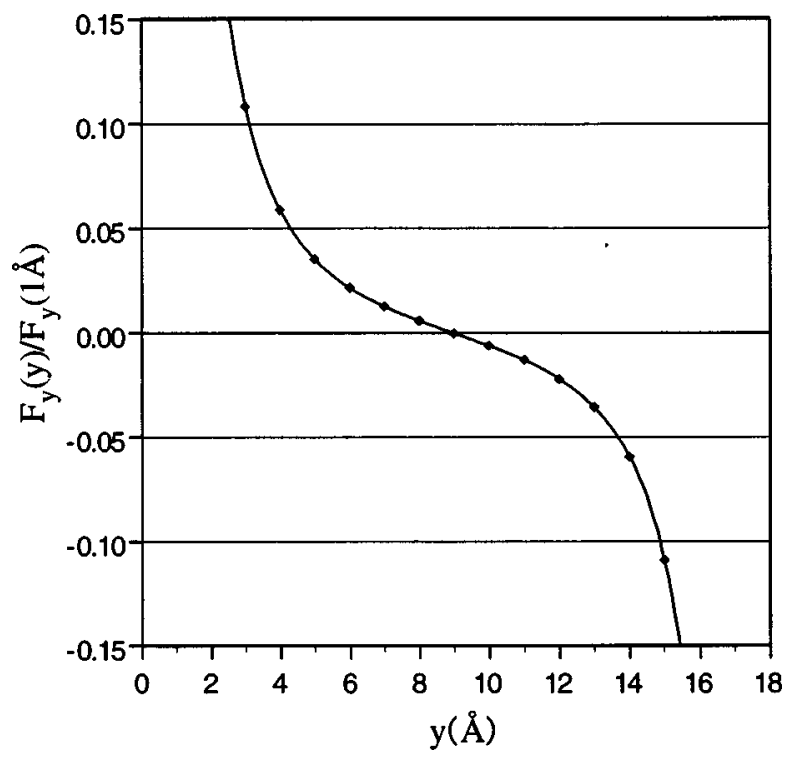

FIG. 2. Comparison of the y-component of force (parallel to the slab surface) acting between two oppositely charged point charges as calculated using EW3DC (line) and $\mathrm{P}^{3} \mathrm{MC}$ (diamonds). The scale is chosen for comparison with Fig. 4 of Ref. 9. 
throughout the occupied half of the simulation cell between the two oppositely charged electrode surfaces, which consisted of single layers of a square lattice of fixed-in-space wall ions centered at $1.5 \AA$ intervals. Each wall ion was assigned LJ parameters of $\sigma_{\mathrm{LJ}}=1.5 \AA$ and $\epsilon / k=50 \mathrm{~K}$, along with a partial charge corresponding to the desired electrode charge density.

Canonical ensemble (NVT) simulations were performed with the temperature set at $25^{\circ} \mathrm{C}$ and the volume chosen to give a bulk density of approximately $60 \mathrm{~mol} / \mathrm{L}$. A somewhat high density was chosen in order to ensure that bulk liquid water would be present in all of the cases without the need to adjust the volume for the individual runs. The simulations were allowed to equilibrate for $50 \mathrm{ps}$ prior to the density profile accumulation period of 200 ps (80000 time steps of 2.5 fs each). Twenty repetitions, each with a unique starting configuration, were performed at each state point of interest in order to obtain adequate ensemble averaging and smooth ion density distribution profiles. Each repetition required approximately $16 \mathrm{~h}$ of CPU time on a 32 node SGI Origin 2000 supercomputer.

\section{RESULTS AND DISCUSSION}

We present in Figs. 3-5 water and ion $z$-direction (perpendicular to the electrode) density distribution profiles for the 12 cases studied at various surface charges, $\sigma$. No ions are present in Fig. 3, and the water density profiles are omitted from Figs. 4 and 5 for clarity. Water density profiles for Figs. 4 and 5 are essentially identical to the water density profiles of Fig. 3 with the corresponding electrode surface charge. In all cases, the positively charged electrode is on the left, and the negatively charged electrode is on the right. Flat, neutral density profiles seen in the central regions of all of the plots indicate the presence of completely shielded bulk electrolyte fluid. The presence of the bulk fluid shows that the positive and negative electrodes are isolated, and enables us to examine the entire shielding layer of fluid at either electrode. ${ }^{2,28}$ The oxygen and hydrogen profiles are quite smooth in the central region, but the ionic profiles are less smooth due to the greater statistical uncertainty. Other authors have also noted this problem.

Even in the absence of ions or electrode surface charge, we see a pronounced difference between bulk and interfacial water characteristics (see Fig. 3, top panel). Other studies confirm this finding, ${ }^{1,24,32-34}$ and point out that the difference is even more pronounced as the electrode is charged. ${ }^{2,18,32-34}$ Especially encouraging is the agreement between these molecular dynamics (MD) results and force measurements of Israelachvili et al. ${ }^{17}$ and the x-ray scattering findings of Toney et al. ${ }^{18}$ They found, contrary to commonly used theories, such as the Gouy-Chapman (GC) theory, ${ }^{35,36}$ that the water is ordered in layers extending several molecular diameters from the electrode. They also observed strong dipole orientation near the charged surfaces. The oxygen and hydrogen water profiles presented here agree, at least qualitatively, on these points. Earlier theoretical studies of electrochemical interfaces using simpler molecular solvents also show this effect. $^{32-34}$

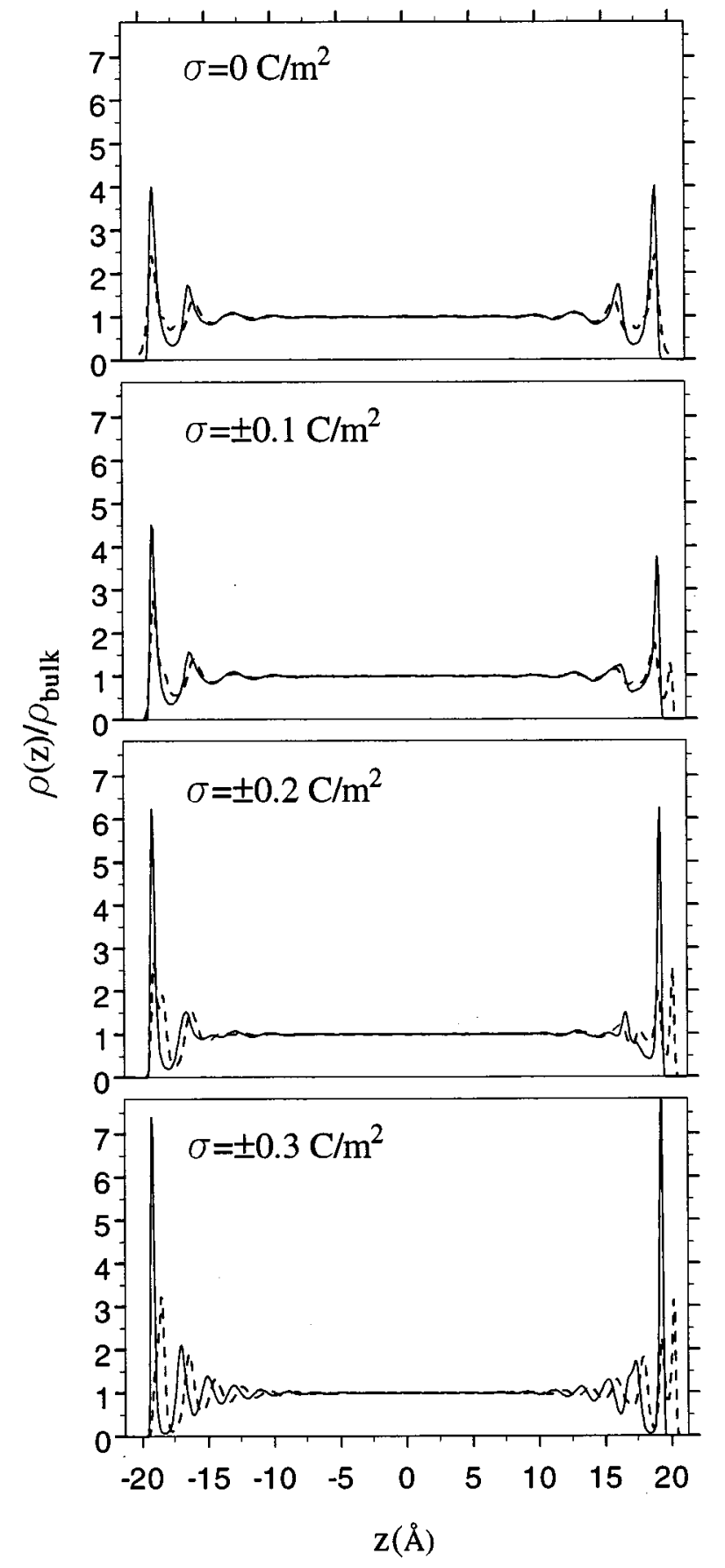

FIG. 3. Oxygen (solid line) and hydrogen (broken line) average density distribution as a function of distance from the center of the simulation cell. The positive and negative electrodes are centered at -21.28 and $21.28 \AA$, respectively. Bulk anion and cation concentrations are $0 \mathrm{M}$.

Despite the generally good agreement between these MD results and the experimental findings of Toney et al. ${ }^{18}$ we also note a disagreement concerning the density of water next to the charged surface. While the experimental findings indicate that the average density of water across the first peak and valley next to the surface is much higher than the bulk water density, our results indicate no such effect. This is in agreement with other MD simulation results ${ }^{37,38}$ even though different electrode models were used. We refer the reader to the article by Yeh and Berkowitz ${ }^{38}$ for a discussion of this discrepancy between the simulation and experimental results. 


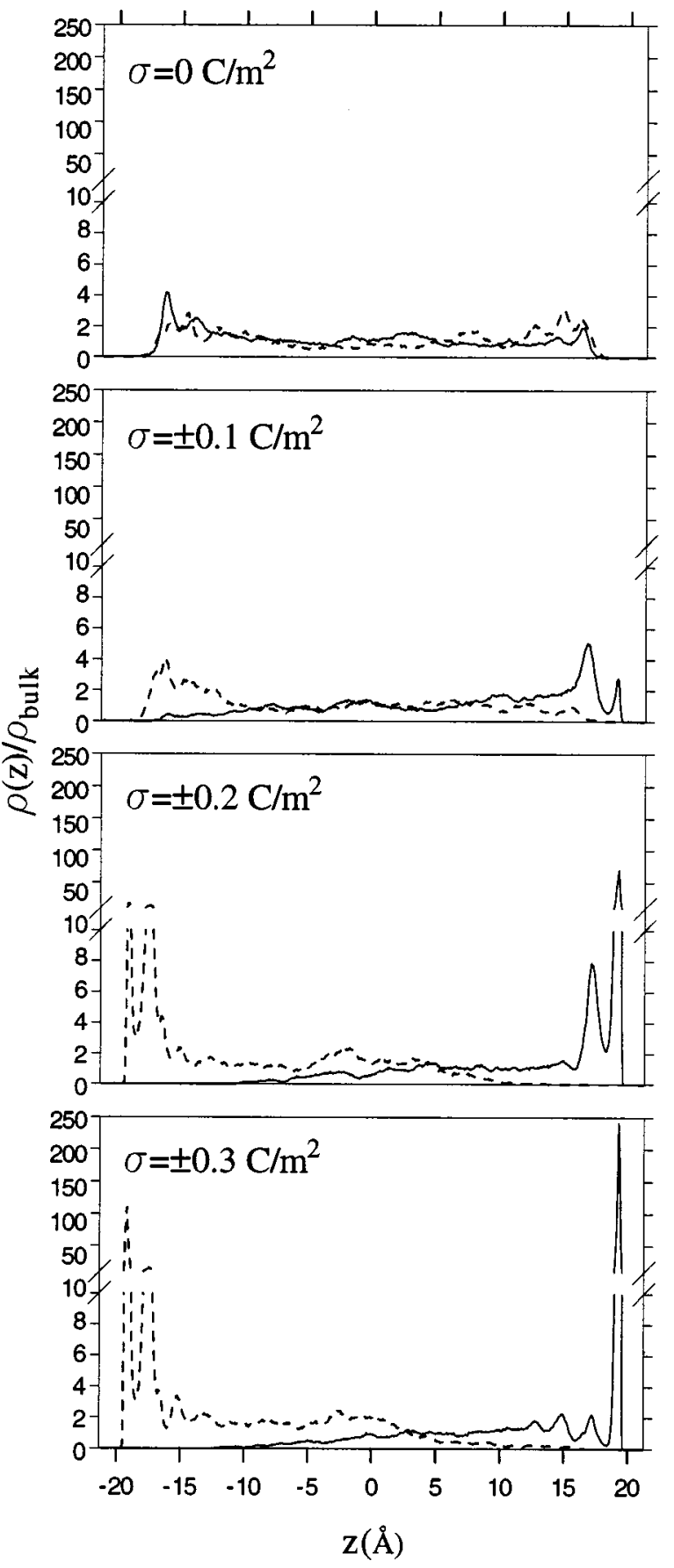

FIG. 4. Cation (solid line) and anion (broken line) average density distribution as a function of distance from the center of the simulation cell. The positive and negative electrodes are centered at -21.28 and $21.28 \AA$, respectively. Bulk anion and cation concentrations are $0.25 \mathrm{M}$.

Without ions present, one might expect a pure solvent to behave similarly at equally and oppositely charged electrodes, but the lack of symmetry of the water molecules about all axes of rotation introduces asymmetry in the density profiles, as can be seen by comparing the left and right hand sides of the plots of Fig. 3.

Surprisingly, the addition of ions does little to change the water density distributions. Except in the extreme case of $\pm 0.3 \mathrm{C} / \mathrm{m}^{2}$, the ions make very small contributions to the charge shielding of the electrode when compared to the contribution of the water molecules. Potential plots at varying
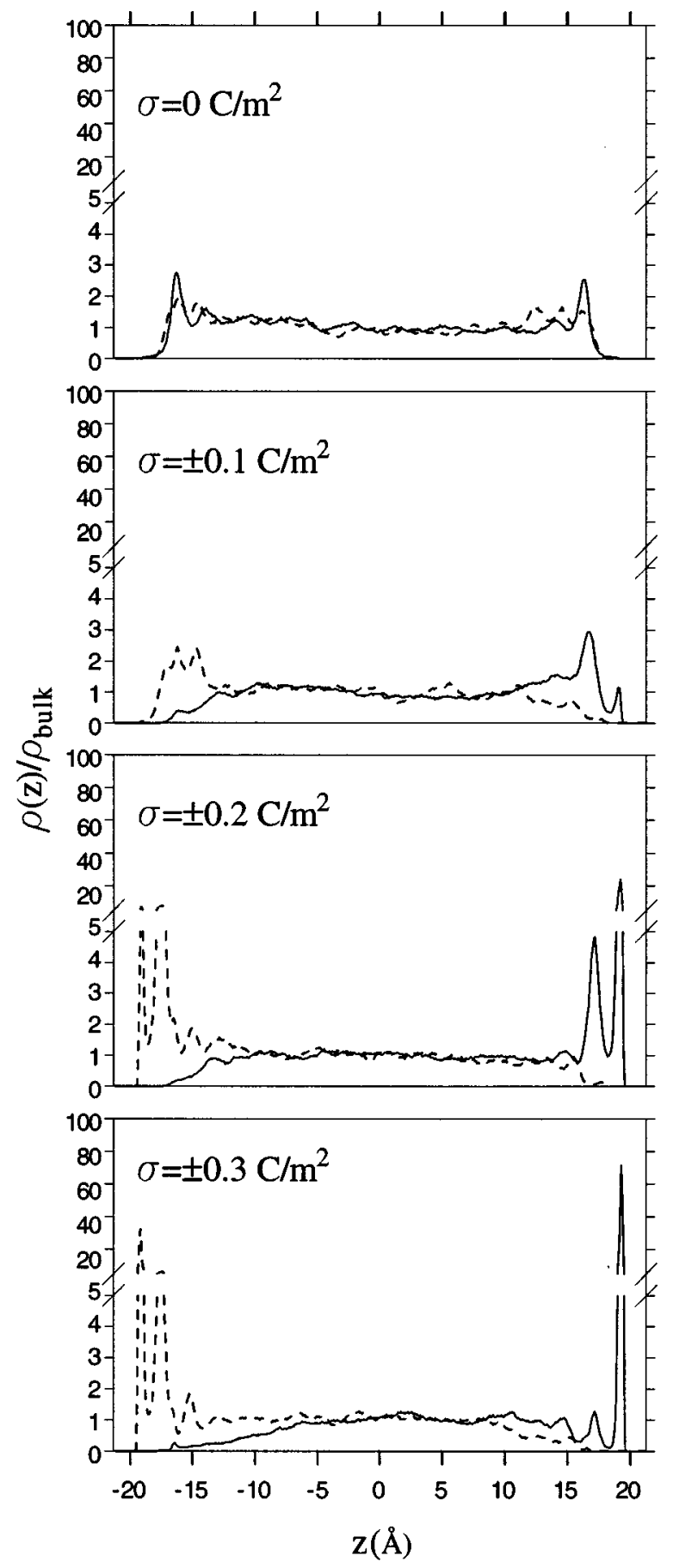

FIG. 5. Cation (solid line) and anion (broken line) average density distribution as a function of distance from the center of the simulation cell. The positive and negative electrodes are centered at -21.28 and $21.28 \AA$, respectively. Bulk anion and cation concentrations are $1 \mathrm{M}$.

ion concentration of the same moderate electrode charge show insignificant differences (see Fig. 6). Only for the physically extreme case of $\pm 0.3 \mathrm{C} / \mathrm{m}^{2}$ is a significant deviation with change in ion concentration observed. It is wellknown from experiment ${ }^{39}$ that the potential and capacitance show strong ion concentration dependence as well as electrode charge dependence at low ionic concentrations. This phenomenon was not observed in our simulations. It may be necessary to go to concentrations well below $0.25 \mathrm{M}$ to ob- 


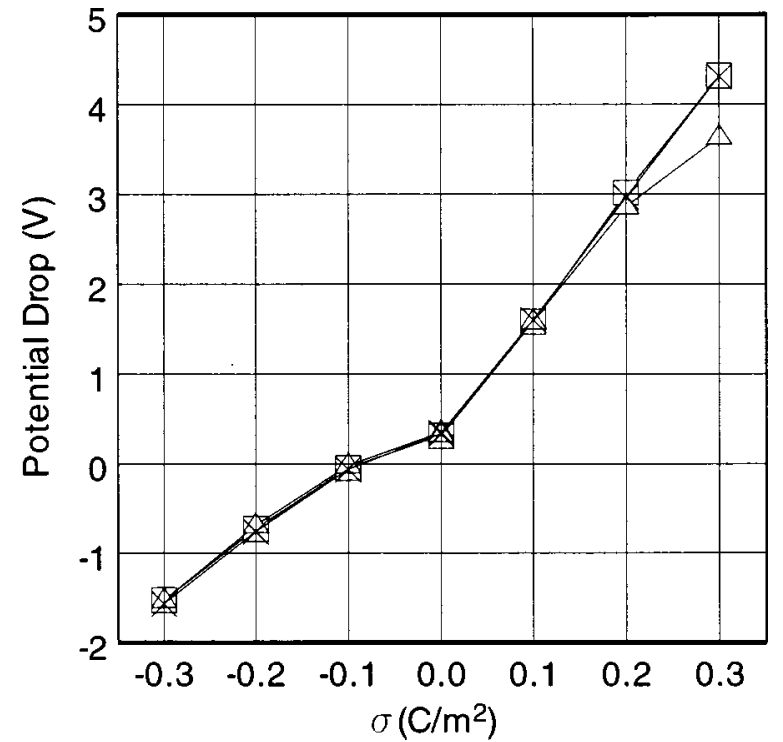

FIG. 6. Potential drop across the double layer (from the middle of the slab to the electrode surface) as a function of surface charge density and bulk ion concentration. The $\triangle, \times$, and $\square$ represent $0,0.25$, and $1 \mathrm{M}$ bulk anion and cation concentrations, respectively.

serve this phenomenon. This, regrettably is exceedingly demanding of computer resources.

It is also well known that an ion's size has a strong influence on its solubility, and hence its adsorptivity onto the surface of an electrode. The results shown here are all for equal-sized ions; the effect of ion size on solvation, surface adsorption, and the ability of the ion to affect the double layer capacitance is the subject of a future study. Here, we are interested in the effects of charge and ion concentration. Even though the size of the cations and anions was chosen to be equal for the purposes of this study, the asymmetric charge distribution of the water molecules causes asymmetric anion and cation behavior. The cations are clearly more easily adsorbed onto the electrode surface, which also suggests better anion solvation, and slower anion transport. At zero charge we observe no wall contact adsorption of either species, and at $\pm 0.3 \mathrm{C} / \mathrm{m}^{2}$ both species are strongly adsorbed to the electrode surfaces. It is expected that this observation may change when the cations and anions have different diameters. The effect of ion size difference will be considered in a subsequent study.

Also evident at large surface charges is the well-known fact that the concentration of ions at a charged electrode can be much larger than the bulk ion concentration. ${ }^{18,19,32-36,40}$ The bottom panes of Figs. 4 and 5, which correspond to an electrode charge of $\pm 0.3 \mathrm{C} / \mathrm{m}^{2}$, show peaks of electrodeadsorbed ions at concentrations of more than two orders-ofmagnitude larger than the corresponding bulk ion concentrations.

Figure 7 shows the potential drop across the double layer as a function of distance from the electrode surface. Theories, such as the GC theory, that do not consider the molecular nature of the solvent, are unable to predict the oscillatory pattern seen here. More sophisticated theories do show this effect. ${ }^{32-34}$ Even in the case of no charge and no ions, this oscillatory pattern is clearly evident as seen in Fig. 7. A

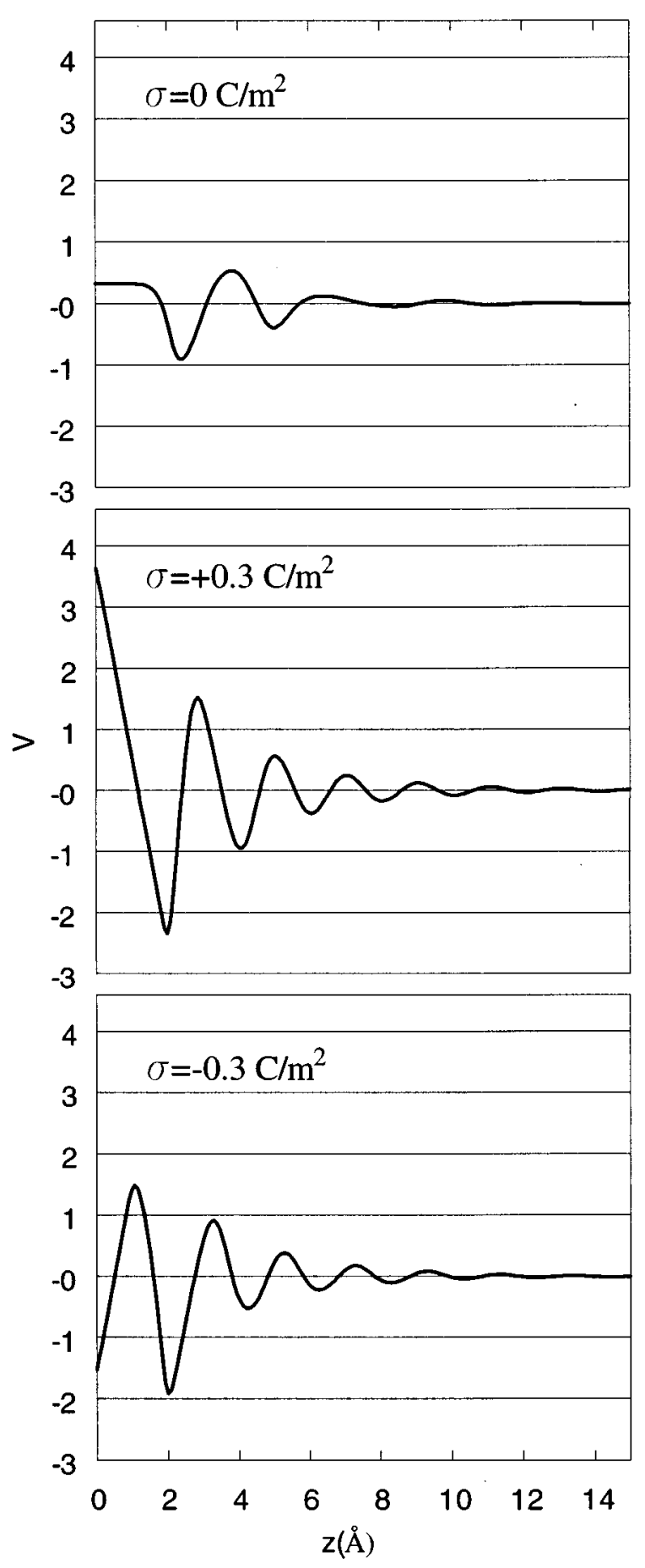

FIG. 7. Potential drop from the neutral bulk fluid to the electrode surface as a function of distance from the electrode surface. Bulk anion and cation concentrations are $0 \mathrm{M}$.

comparison between the bottom two panes of Fig. 7 is also noteworthy, since it demonstrates how the asymmetric nature of the solvent molecules dramatically affects the potential drop across the double layer. Even though the electrode surface charge is equal and opposite, the plots are radically different. Although the potential drop across the double layer seems to be only mildly affected by ion concentration, it is strongly affected by electrode charge (see Fig. 6). 


\section{CONCLUSIONS}

The $\mathrm{P}^{3} \mathrm{MC}$ method is a well-defined and efficient way to model coulombic interactions for large slab geometry electrolytic systems. Its flexibility and adaptability make it an attractive way to properly account for long-range intermolecular interactions at the accuracy level and speed that the user desires. This method has enabled us to accurately perform these MD calculations within a reasonable amount of computer time.

Unlike many previous double layer simulations, the molecular nature of the solvent was not neglected here, and it was found that the water molecules were responsible for most of the potential drop across the double layer and that ion concentration had little effect.

Due to the charge distribution and shape of the water molecule, very different behavior was observed at equally and oppositely charged electrodes. With the models used here, the cations more easily adsorbed to the electrode surface than the anions, which indicates better solvation of the anions by the water molecules. Given sufficient electrode charge, contact adsorption of either species occurred. In fact, at very high charge, the ion concentration profile shows a peak at the electrodes in excess of 100 times the value of the bulk ion concentration. Overall, results of this MD study were in good qualitative agreement with experimental findings, and those found in earlier, less sophisticated theoretical results.

\section{ACKNOWLEDGMENTS}

This work was supported in part by the National Science Foundation (Grant No. CHE98-13729) and by the donors of the Petroleum Research Fund, administered by the American Chemical Society (Grant No. ACS-PRF 31573-AC9).

${ }^{1}$ C. Y. Lee, J. A. McCammon, and P. J. Rossky, J. Chem. Phys. 80, 4448 (1984).

${ }^{2}$ S. H. Lee and P. J. Rossky, J. Chem. Phys. 100, 3334 (1994).

${ }^{3}$ J. N. Glosli and M. R. Philpott, J. Chem. Phys. 96, 6962 (1992).

${ }^{4}$ D. A. Rose and I. Benjamin, J. Chem. Phys. 95, 6856 (1991).

${ }^{5}$ D. A. Rose and I. Benjamin, J. Chem. Phys. 98, 2283 (1993).
${ }^{6}$ B. Eck and E. Spohr, Electrochim. Acta 42, 2779 (1997).

${ }^{7}$ E. Spohr, J. Chem. Phys. 107, 6342 (1997).

${ }^{8}$ I.-C. Yeh and M. L. Berkowitz, J. Chem. Phys. 111, 3155 (1999).

${ }^{9}$ P. S. Crozier, R. L. Rowley, E. Spohr, and D. Henderson, J. Chem. Phys. 112, 9253 (2000).

${ }^{10}$ R. W. Hockney and J. W. Eastwood, Computer Simulation Using Particles (Institute of Physics, Bristol, 1988).

${ }^{11}$ T. Darden, D. York, and L. Pedersen, J. Chem. Phys. 98, 10089 (1993).

${ }^{12}$ B. A. Brock, G. T. Tironi, and W. F. van Gunsteren, J. Chem. Phys. 103, 3014 (1995).

${ }^{13}$ U. Essmann, L. Perera, M. L. Berkowitz, T. Darden, H. Lee, and L. G. Pedersen, J. Chem. Phys. 103, 8577 (1995).

${ }^{14}$ M. Deserno and C. Holm, J. Chem. Phys. 109, 7678 (1998).

${ }^{15}$ M. Deserno and C. Holm, J. Chem. Phys. 109, 7694 (1998).

${ }^{16}$ E. L. Pollock and J. Glosli, Comput. Phys. Commun. 95, 93 (1996).

${ }^{17} \mathrm{~J}$. N. Israelachvili, Intermolecular and Surface Forces, 2nd ed. (Academic, London, 1992).

${ }^{18}$ M. F. Toney, J. N. Howard, J. Richer, G. L. Borges, J. G. Gordon, O. R. Melroy, D. G. Wiesler, D. Yee, and L. B. Sorensen, Surf. Sci. 335, 326 (1995).

${ }^{19}$ G. M. Torrie and J. P. Valleau, J. Chem. Phys. 73, 5807 (1980).

${ }^{20}$ D. Boda, K.-Y. Chan, and D. Henderson, J. Chem. Phys. 109, 7362 (1998).

${ }^{21}$ J. A. Barker and R. O. Watts, Chem. Phys. Lett. 3, 144 (1969).

${ }^{22}$ R. O. Watts, Mol. Phys. 28, 1069 (1974).

${ }^{23}$ N. I. Christou, J. S. Whitehouse, D. Nicholson, and N. G. Parsonage, Mol. Phys. 55, 397 (1985).

${ }^{24}$ S.-B. Zhu and G. W. Robinson, J. Chem. Phys. 94, 1403 (1991).

${ }^{25}$ D. E. Parry, Surf. Sci. 49, 433 (1975); 54, 195 (1976).

${ }^{26}$ D. M. Heyes, M. Barber, and J. H. R. Clarke, J. Chem. Soc., Faraday Trans. 2 73, 1485 (1977).

${ }^{27}$ S. W. de Leeuw and J. W. Perram, Mol. Phys. 37, 1313 (1979).

${ }^{28}$ L. Greengard and V. Rokhlin, J. Comput. Phys. 60, 187 (1985).

${ }^{29}$ M. R. Philpott and J. N. Glosli, J. Electrochem. Soc. 142, L25 (1995).

${ }^{30}$ F. Figueirido, R. M. Levy, R. Zhou, and B. J. Berne, J. Chem. Phys. 106, 9835 (1997).

${ }^{31}$ H. J. Berendsen, J. R. Grigera, and T. P. Straatsma, J. Phys. Chem. 91, 6269 (1987).

${ }^{32}$ L. Blum and D. Henderson, J. Chem. Phys. 74, 1902 (1981).

${ }^{33}$ S. L. Carnie and D. Y. C. Chan, J. Chem. Phys. 73, 2949 (1980).

${ }^{34}$ D. Henderson, F. F. Abraham, and J. A. Barker, Mol. Phys. 31, 1291 (1976).

${ }^{35}$ G. Gouy, J. Phys. (Paris) 9, 457 (1910).

${ }^{36}$ D. L. Chapman, Philos. Mag. 25, 475 (1913).

${ }^{37}$ K. J. Schweighofer, X. Xia, and M. L. Berkowitz, Langmuir 12, 3747 (1996).

${ }^{38}$ I.-C. Yeh and M. L. Berkowitz, Chem. Phys. Lett. 301, 81 (1999).

${ }^{39}$ D. C. Grahame, J. Chem. Phys. 21, 1054 (1953).

${ }^{40}$ I. Danielewicz-Ferchmin, A. R. Ferchmin, and A. Szlaferek, Chem. Phys. Lett. 288, 197 (1998). 